\title{
UPLC-Q-TOF/MS based Metabolomics Approach to reveal the hepatotoxicity of emodin and detoxification of Dihydromyricetin
}

Jian $\mathrm{Gao}^{\# 1,2}$, Ning Shi ${ }^{\# 3}$, Hongju Guo ${ }^{3}$, Junfeng $\mathrm{Gao}^{2}$, Xu Tang${ }^{2}$, Siyuan Yuan², Jiahui Qian ${ }^{1}$, Binyu Wen*2

1. Beijing University of Chinese Medicine, Beijing, 100029, P.R.China

2. Dongfang Hospital, Beijing University of Chinese Medicine, Beijing, 100078, P.R.China

3. Pharmaceutical Department of Characteristic Medical Center, Strategic Support Force, Beijing, 100101, P.R.China

\#These authors contributed equally to this work.

*Correspondence: Binyu Wen E-mail: wen-binyu@163.com 

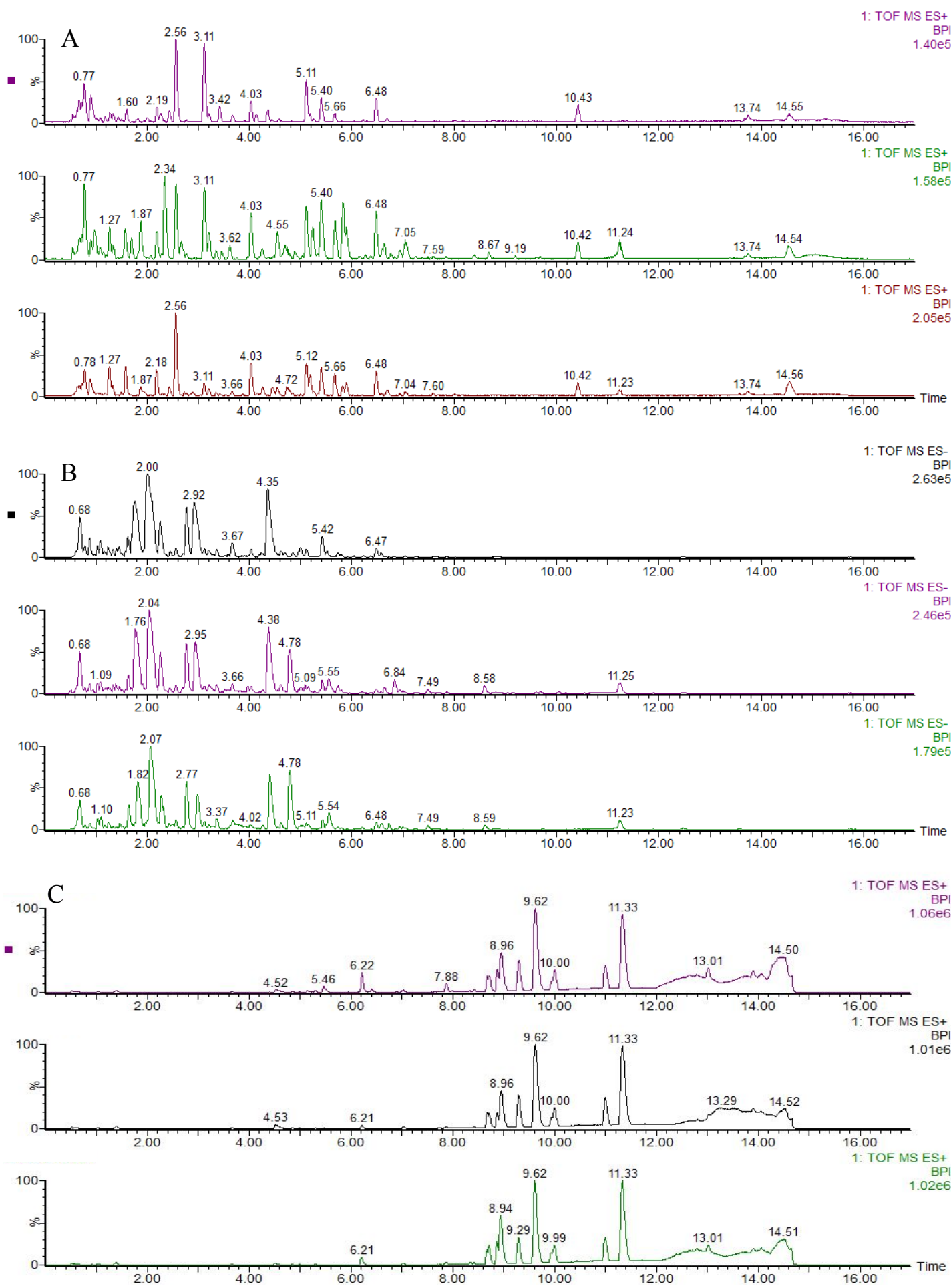

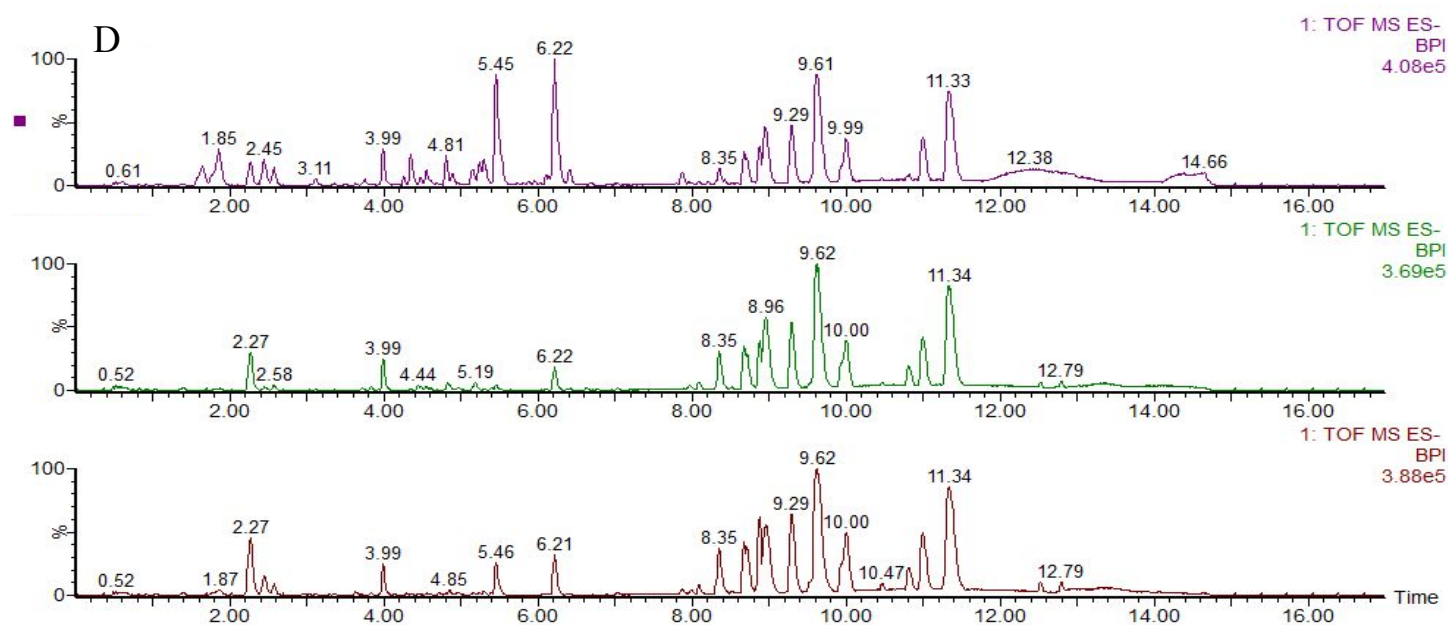

Figure S1. UPLC-Q-TOF-MS representative base peak intensity(BPI)

chromatogram of the positive and negative ions in urine and serum samples

The peaks were acquired via analyses of urine and serum samples of different groups in urine $\mathrm{ESI}^{+}(\mathrm{A})$, urine $\mathrm{ESI}^{-}(\mathrm{B})$ modes, serum $\mathrm{ESI}^{+}(\mathrm{C})$ and serum $\mathrm{ESI}^{-}$(D) modes. $\mathrm{ESI}^{+}$and $\mathrm{ESI}^{-}$from top to bottom show the Con group, emodin group, and emodin+DMY group. 